\title{
Attitude Over Knowledge and Skill: Enabler of Research Productivity (RP) Among Graduate Students in One State University in the Philippines
}

\author{
Adriel G. Roman \\ adriel.roman@1spu.edu.ph \\ Laguna State Polytechnic University, L. de Leon St. Siniloan (4019), Laguna, Philippines
}

\begin{abstract}
The aim of this study is to measure the research knowledge, skills, and attitudes (KSA) of graduate students and test whether the KSA are enablers of research productivity (RP) of graduate students. A descriptive-correlation research design of the research was employed with fifty purposively selected graduate students served as the respondents of the study. A validated research instrument consisting of 121 items was utilized with an average Cronbach's alpha of 0.967. The questionnaire was administered before the classes in Advanced methods of research begins. Based on the study, the level of knowledge and skills of the graduate students were on the average level while their attitude was high. It was also found that the research productivity of graduate students considering the completed research, presented, published, and research with citations was low. Results of the study showed that attitude affects significantly research productivity. Problems on research writing of the graduate students based on the results include knowledge on statistical analysis, confidence, instrument preparation, among others. Several trainings requested by the respondents are included in the paper.
\end{abstract}

Keywords:Research Attitudes; Research Productivty; Graduate Students

\section{Introduction}

The Philippine Graduate Education showed great importance to research based on the revised policies, standards, and guidelines for graduate programs issued by the Commission on Higher Education (CHEd) which provide an avenue for the cultivation of research culture and innovation in the graduate programs [1]. One of the salient changes in the graduate studies both in Master's and Doctorate by Academic Track and by Research is the publication requirement. This publication requirement has been implemented in the Philippine graduate education programs since 2020. In this requirement, before a graduate student who is enrolled in the graduate program by academic track or by research, graduates, the student must have at least one (1) publication in a refereed journal.

This change in graduate education unlocks opportunities for higher education institutions (HEIs) offering graduate programs to further enhance the research culture in graduate school. This may be done through providing research productivity enhancement training, and research capability training in both faculty and students. Since that research has been one of the core functions of the higher education institutions in the Philippines, faculty in the HEI are expected to produce research [2]-[4]. In support, HEIs, particularly, State Universities and Colleges (SUCs), allot budget for research undertakings comprising $10 \%$ of the total collected tuition fees [5] aside from the different research funding sources that provide research grants. 
However, while this initiative promotes advanced studies for graduate education, the existing research status of the Philippines remains a challenge as compared to other ASEAN countries when it comes to research productivity and impact [6]. In fact, in the study conducted by [7], it was concluded that there are only a few citations of Philippine works in terms of research outputs and that self-citation is becoming a culture of research.

While the Philippine Higher Education sector provides a large commitment to enhancing the capacity and capability of graduate students in research, there is still a need to upgrade the research productivity in the Philippine HEIs [8]. Similarly, several factors were identified as drivers of research performance in HEIs such as knowledge and skills [9], concrete research foundation [10], attitude [11], time management [12], selfefficacy [13], motivation [14], and university interventions [15].

For this reason, research culture in graduate studies must be improved. In light of this, there is a need to study the research competencies of graduate students for a further enhanced graduate studies research capability undertaking. With this aim, the research knowledge, skills, and attitudes of graduate students were examined as enablers of research enhancement in the graduate program. Research KSA plays a significant role in the research productivity of faculty in the institutions of higher learning, thus, this study was conducted.

\section{Objectives}

This study aims to measure the research knowledge, skills, and attitudes (KSA) of graduate students and test whether the KSA are enablers of research productivity (RP) of graduate students. In particular, it sought answers on the following: 1. What is the level of research knowledge and skills of the graduate students based on the competency test? 2. What is the extent of attitude towards the research of the graduate students? 3. What is the research productivity of the graduate students? 4. Will there be any significant predictor(s) of research productivity among graduate students based on their research knowledge and skills, and attitudes?

\section{Methodology}

In pursuit to measure the implication of research KSA to research productivity, the researcher used a descriptive-correlation research design. In this design, the KSA of graduate students were determined using a validated instrument consisting of 121 items segregated as follows: 75 items for measuring the research knowledge and skills of the graduate students (passed the item analysis procedures both item difficulty index and index of discrimination); 40 items for research attitudes (Cronbach's alpha: 0.967); 4 items for research performance as measured by the number research completed, presented, published, and citations while the last 2 items are for problems encountered and preferred research training if given the opportunity. The instrument was administered online to fifty graduate students (Master of Arts in Education with different specializations) who were purposively selected. The fifty respondents were enrolled in Advanced Methods of Research class in one state university. The study was conducted during the second semester of 2021. Respondents were asked for voluntary participation in the research by providing their consent to the researcher. Respondents were assured that all data collected would be treated collectively and highest ethical considerations would be observed. After the data have been collected and analyzed, the results were presented to the respondents in one focus group discussion. Statistical treatments such as arithmetic mean, weighted mean, mode, and regression were utilized. Alpha value was set to $5 \%$. 


\section{Results and Discussion}

\subsection{Level of research knowledge and skills of the graduate students}

The level of research knowledge and skills of the graduate students are presented below. It can be seen from the table that both the research knowledge and skills of the graduate students are on the average level. These results show that before entering the advanced methods of research subject in the graduate studies, respondents are not novices when it comes to research topics. In particular, research concepts in terms of instrumentations and research types both exceed $50 \%$ of the mean percentage scores of the respondents while $27.60 \%$ is the mean percentage of the concept about providing interpretation, conclusion, and recommendations. The result of the study can be attributed to the respondents' previous research experiences during their undergraduate degree in which one of the requirements is to produce collaborative or individual research. In addition, since the majority of the respondents are also in the teaching profession, research activities in their respective posts provide opportunities for them to obtain knowledge and skills in research. Instrumentation in research deals with how to conceptualize research questionnaires and other tools and likewise on the process of determining the validity, reliability, and usability of the developed instruments. The result in terms of this research concept can be related to the type of respondents of the study. For the reason that majority in the graduate school setting where the study was conducted are teachers, they are equipped and much acquainted on preparations of teaching tools and have the tools undergone validation process, for instance, examination. These experiences of the teachers would greatly influence their knowledge and skills in research instrumentations.

Table 1. Level of research knowledge and skills of the graduate students

\begin{tabular}{|c|c|c|c|c|c|}
\hline Competency Test & Mean & \multicolumn{2}{|c|}{$\begin{array}{l}\text { Standard } \\
\text { Deviation }\end{array}$} & \multicolumn{2}{|c|}{$\begin{array}{c}\text { Verbal } \\
\text { Interpretation }\end{array}$} \\
\hline $\begin{array}{l}\text { Research Knowledge } \\
\text { (39 items) }\end{array}$ & 19.804 & \multicolumn{2}{|c|}{4.84} & \multicolumn{2}{|c|}{ Average Level } \\
\hline $\begin{array}{c}\text { Research Skills } \\
\text { (36 items) }\end{array}$ & 15.118 & \multicolumn{2}{|c|}{4.35} & \multicolumn{2}{|c|}{ Average Level } \\
\hline Research Topics & & Mean & $\begin{array}{l}\text { No of } \\
\text { items }\end{array}$ & Percentage & Rank \\
\hline Instrumentations & & 5.10 & 9 & 56.67 & 1 \\
\hline Research Types & & 3.74 & 7 & 53.43 & 2 \\
\hline Data Analysis Procedure & & 4.30 & 9 & 47.78 & 3 \\
\hline Theories and Framework & & 2.82 & 6 & 47.00 & 4 \\
\hline Research Problems & & 4.14 & 9 & 46.00 & 5.5 \\
\hline Literatures, Sources and & d Citations & 3.22 & 7 & 46.00 & 5.5 \\
\hline Research Methods & & 3.66 & 8 & 45.75 & 7 \\
\hline Sampling Techniques & & 3.86 & 9 & 42.89 & 8 \\
\hline Research Ethics & & 2.08 & 6 & 34.67 & 9 \\
\hline $\begin{array}{l}\text { Interpretation, } \\
\text { Recommendation }\end{array}$ & Conclusion, & 1.38 & 5 & 27.60 & 10 \\
\hline
\end{tabular}


4.2. Extent of attitude towards research of the graduate students based on Research beliefs; Values; Time and personal resources; and Practices

Research attitudes of the respondents were divided into four major constructs namely resources, beliefs, values, and practices. Based on the results, graduate students have a high extent of attitudes toward research. As seen also, three constructs, resources, beliefs, and values are on the high extent while the construct about practices is to a moderate extent. Results revealed that graduate students have a positive attitude towards research that they devote specific time in doing research even at home. They also provide themselves with materials for research purposes. They also tap other people to assist them in their research activities in school. For the beliefs part of the analysis, results reveal that graduate students are on the positive thought that research improves the quality of teaching and is an important part of their professional growth. They are also on the positive thought that they possess the good qualities of being a researcher. The high extent of the research values is based on the graduate students' fairness, compassion, commitment, openness, objectivity, and fairness.

Table 2. Extent of attitude towards research of the graduate students based on Research beliefs; Values; Time and personal resources; and Practices

\begin{tabular}{lll}
\hline Attitude & Mean & Interpretation \\
\hline Resources & 3.729 & High Extent \\
Belief & 4.186 & High Extent \\
Values & 4.164 & High Extent \\
Practices & 3.329 & Moderate Extent \\
Weighted Mean & 3.852 & High Extent \\
\hline
\end{tabular}

\subsection{Research productivity of the graduate students}

The research productivity of the graduate students as measured in terms of number of completed research, number of the presented research, number of published research, and number of published research with citations is presented. The results reveal that 14 out of 50 graduate students have completed research aside from their undergraduate theses. Out of 14, twelve are able to present their completed research to research forums/conferences. Eight graduate students responded that they have research publications in refereed journals while 3 out of fifty respondents have already their citations. The results about the research outputs of the graduate students provide evidence that the research outputs produced are not sounding before they take the advanced research methods class in the graduate program/school. The result of the analysis provides opportunities for the graduate school administration to strengthen its research program since the majority of the graduate students have minimal research outputs.

Table 3. Research productivity of the graduate students

\begin{tabular}{lllllll}
\hline Research & & & & & & \\
Productivity & With & $\%$ & Without & $\%$ & Total & $\%$ \\
\hline Completed & 14 & 28.00 & 36 & 72.00 & 50 & 100.00 \\
Presented & 12 & 24.00 & 38 & 76.00 & 50 & 100.00 \\
Published & 8 & 16.00 & 42 & 84.00 & 50 & 100.00 \\
Cited & 3 & 6.00 & 47 & 94.00 & 50 & 100.00 \\
\hline
\end{tabular}




\subsection{Enabler of research productivity among graduate students}

The knowledge, skills, and attitudes of the graduate students were tested if there is at least one that is an enabler of the graduate student's research productivity. Before using the regression test, the normality test using Shapiro-Wilk was done for the independent variables and the dependent variable. Based on the SWNormality test, all independent variables (knowledge, skills, attitudes) passed the normality test (Knowledge: SW=0.450, $\mathrm{p}=0.421$; Skills: $\mathrm{SW}=0.955 ; \mathrm{p}=0.635$; and Attitude: $\mathrm{SW}=0.977, \mathrm{p}=0.955$ ). For the research productivity of the graduate students, the research employed the formula $\mathrm{RP}=1+0.25 \mathrm{Co}+0.25 \mathrm{Pr}+0.25 \mathrm{Pu}+0.25 \mathrm{Ci}$ where $\mathrm{Co}=$ Completed, $\mathrm{Pr}=$ Presented, $\mathrm{Pu}=\mathrm{Published}$, and $\mathrm{Ci}=$ Research with Citation. The result then was tested for normality using Shapiro-Wilk and likewise passed $(\mathrm{SW}=0.934, \mathrm{p}=0.351)$. The regression result revealed that among the three independent variables considered, only attitudes showed a significant effect on the research productivity of the graduate students while knowledge and skills of the graduate students showed no significant effects on the research productivity of the graduate students. To determine further the determinants of significance in the attitude, regression analysis for each of the constructs was done. Based on the results, beliefs, values, and practices significantly affect the research productivity of graduate students while resources did not show a significant effect. In addition, $50.69 \%$ of the variation of research productivity of the graduate students is attributed to their overall attitudes towards research. Attitude is very important in a researcher's journey. Either a positive or negative attitude towards research can result in sound research productivity or a loss of motivation.

Table 4. Enabler of research productivity among graduate students

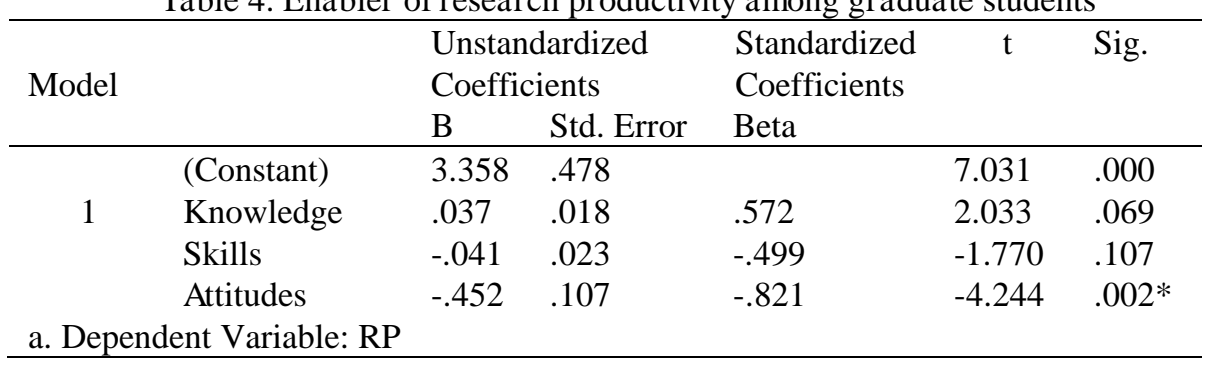

\begin{tabular}{lcccc}
\hline Models & $\mathrm{r}$ & $\mathrm{r}^{2}$ (in\%) & $\mathrm{p}$-value & Regression \\
\hline Overall (Attitude) & 0.712 & $50.69 \%$ & 0.004 & $\mathrm{RP}=3.278-0.392 \mathrm{~A}$ \\
Resources $\left(\mathrm{A}_{1}\right)$ & 0.525 & $27.56 \%$ & 0.054 & Not Significant \\
Belief $\left(\mathrm{A}_{2}\right)$ & 0.614 & $37.70 \%$ & 0.019 & $\mathrm{RP}=3.352-0.345 \mathrm{~A}_{2}$ \\
Values $\left(\mathrm{A}_{3}\right)$ & 0.630 & $39.69 \%$ & 0.016 & $\mathrm{RP}=3.552-0.395 \mathrm{~A}_{3}$ \\
Practices $\left(\mathrm{A}_{4}\right)$ & 0.679 & $46.10 \%$ & 0.008 & $\mathrm{RP}=3.005-0.330 \mathrm{~A}_{4}$ \\
\hline
\end{tabular}

\subsection{Problems Encountered in Research Writing}

Eight problems were enumerated based on the responses of the graduate students. Problems include lack of knowledge on doing research, statistical treatment of data, funding, confidence, difficulties on literature searching, research methods, constructing research title, and time constraints. Respondents identified that lack of knowledge on doing research is the main problem that they experience. Based on the respondents' comments, they did not have any research during their undergraduate studies thus, they do not have any idea on how to start doing research, however, they are very positive about doing research. 
Table 5. Problems Encountered in Research Writing

\begin{tabular}{llc}
\hline \multicolumn{2}{l}{ Problems } & Frequency \\
\hline 1. & Lack of Knowledge on Doing Research & 6 \\
2. & Lack of Knowledge on Statistical Treatment of Data & 5 \\
3. & Time Constraints & 5 \\
4. & Difficulties on Constructing Research Title & 3 \\
5. & Lack of Funding & 2 \\
6. & Lack of Confidence & 1 \\
7. & Difficulties on Literature Searching & 1 \\
8. & Difficulties on Research Methods & 1 \\
Total & & 24 \\
\hline
\end{tabular}

\subsection{Requested Research Training}

Training about doing action research is the most requested activity of graduate students. It is followed by research training on teaching during the time of pandemic. Other training requested by the graduate students include assessment of students' learning, experimental research, language research, instrument preparation, research methodology, and statistical treatment of data.

Table 6. Requested Research Training

\begin{tabular}{clc}
\hline \multicolumn{2}{c}{ Training Requested } & Frequency \\
\hline 1. Action Research & 3 \\
2. $\quad$ Teaching in the time of pandemic & 2 \\
3. Title Construction & 2 \\
4. Assessment of Students Learning & 1 \\
5. Experimental Research & 1 \\
6. Language Research & 1 \\
7. $\quad$ Research Instrument Preparation & 1 \\
8. $\quad$ Research Methods & 1 \\
9. Statistical Treatment for Quantitative Data & 1 \\
Total & 13 \\
\hline
\end{tabular}

\section{Conclusion and Recommendations}

Graduate students have an average level of research knowledge and skills while a high extent of attitude towards research. However, the research productivity of the graduate students is below the average level, and only $20 \%$ percent are publishing papers in refereed research journals. This study concluded that among research knowledge, skills, and attitude (KSA), attitude affects the research productivity of the graduate students in particular their research beliefs, values, and practices. Research beliefs, values, and practices of the graduate students strongly justified their level of research productivity. For these reasons, it is suggested that the requested training of the graduate students be provided to them to further equip themselves with the needed competencies to achieve higher research productivity. 


\section{References}

[1] https://ched.gov.ph/wp-content/uploads/CMO-No--15-Series-of-2019-\%E2\%80\%93-PoliciesStandards-and-Guidelines-for-Graduate-Programs-Updated.pdf

[2] Cocal, C., Cocal E.,\& Celino, B. (2017). Factors Limiting Research Productivity of Faculty Members of a State University: The Pangasinan State University Alaminos City Campus Case. Asia Pacific Journal of Academic Research in Social Sciences, 2 (43-48).

[3] Mantikayan, J. M., \& Abdulgani, M. A. (2018). Factors Affecting Faculty Research Productivity: Conclusions from a Critical Review of the Literature. JPAIR Multidisciplinary Research, 31(1).

[4] https://ched.gov.ph/wp-content/uploads/2017/10/CMO-No.46-s2012.pdf

[5] https://ched.gov.ph/wp-content/uploads/2017/10/CMO-No.20-s2011.pdf

[6] https://www.topuniversities.com/university-rankings/asian-university-rankings/2022

[7] Guido, R. M. D., \& Orleans, A. V. (2020). Philippine Research Productivity in Education Research: A Comparative Performance in Southeast Asia. Asia Pacific Journal of Multidisciplinary Research, 8(4).

[8] Roman, A. G. (2021). Research Competencies and Performance of Higher Education Institutions (HEI) Faculty. International Joournal of Research Publications, 78(1), 8-8. doi:10.47119/IJRP100781620211975

[9] Agatep, J.L.E. \& Villalobos, R.N. (2020) Research capabilities among selected graduate school students in Philippines. Science Insights Education Frontiers, 6(2):691-705

[10] Bueno, D.C. (2019). Research and publish or hibernate? Analysis of the limiting factors towards scientific productivity among professorial lecturers in the Philippines. CC The Journal: A Multidisciplinary Research Review, Volume 14, (October 2019). ISSN 1655-3713.

[11] Shahzad, S., Khalid, M., Farooqi, S. M., \& Maryam, H. (2018). Correlation of University Teachers' Attitude towards Research and their Research Productivity. Al-Qalam, 23(1.), 523-535.

[12] Villalino, N. A. P., \& Cagasan, E. G. (2012). Conditions surrounding publication performance of faculty members of two selected higher education institutions in Eastern Visayas, Philippines. Annals of Tropical Research, 34, 75-94.

[13] Mantikayan, J. M., \& Abdulgani, M. A. (2018). Factors affecting faculty research productivity: Conclusions from a critical review of the literature. JPAIR Multidisciplinary Research, 31(1), 1-21.

[14] Narbarte, M. P. (2018). Research Involvement, Motivation, and University Initiatives as Agents for Enhancing Research Culture and Quality. Human Behavior, Development and Society, 17, 68-78.

[15] Quitoras, M. C. L. \& Abuso, J. E. (2021). Best Practices of Higher Education Institutions (HEIs) for the Development of Research Culture in the Philippines. Pedagogical Research, 6(1), em0087. https://doi.org/10.29333/pr/9355 